\title{
Emprendimientos turísticos sustentables: Una revisión de la literatura
}

\author{
Yolanda Mariana Garcia Carranza \\ Ym.garcia.2016@alumnos.urjc.es
}

Evelyn Karina Garcia Carranza

Evelyn.garciac@ug.edu.ec

\section{RESUMEN}

\section{Introducción}

En los últimos veinte años, el emprendimiento se ha convertido en la alternativa que tienen los gobiernos para el desarrollo de sus economías y disminución del desempleo como medida que aumenta el bienestar social sobre todo en periodos de crisis y recesiones económicas. Los emprendimientos turísticos parte de la necesidad de una población o región de satisfacer necesidades de turistas nacionales o extranjeros. El turismo sostenible o turismo sustentable busca minorar el impacto que tienen las actividades turísticas al medioambiente y a las culturas locales de una región. El objetivo de la presente investigación consiste en analizar los aportes en el campo científico y académico de los emprendimientos en el sector turístico en el contexto de la sustentabilidad con el entorno. La metodología utilizada fue de tipo descriptiva- cuantitativa a través de un análisis bibliográfico se escogió la plataforma Web of Science (W.O.S.) por contener un alto número de revista de calidad e impacto en la comunidad científica en las áreas de ciencias sociales, se obtuvieron 69 artículos en la colección principal que representan documentos de acceso abierto con el criterio de búsqueda "tourism entrepreneurship" AND "sustainable tourism" desde el año 2008 hasta el 2020. Los principales resultados dieron a conocer que el primer artículo que vincula esta temática surge a partir del año 2008 aunque es desde el 2016 que incrementa el interés en las investigaciones, centrándose en las áreas de tecnología de ciencia sostenible verde y estudios ambientales. Los principales autores con aportaciones científicas le corresponden a Xavier Font y Marianna Sigala sus estudios se basan en marketing sustentable en el turismo y hospitalidad turística. Inglaterra, España y Romania son los países con mayores contribuciones. La principal conclusión determina que en los países europeos son las pequeñas empresas las que se 
encuentran más motivadas para ser sostenibles, la gran mayoría de estos emprendimientos desenvuelven su actividad en espacios naturales protegidos y creen que el apoyo de instituciones públicas y privadas es vital para el desarrollo de actividades económicas buscando la conservación natural del planeta.

Palabras claves: emprendimiento; emprendimiento turístico; turismo sustentable. 


\title{
Sustainable tourism ventures: A review of the literature
}

\begin{abstract}
In the last twenty years, entrepreneurship has become the alternative that governments have for the development of their economies and the reduction of unemployment as a measure that increases social well-being, especially in periods of economic crisis and recessions. Tourist undertakings are based on the need of a population or region to satisfy the needs of national or foreign tourists. Sustainable tourism or sustainable tourism seeks to reduce the impact that tourism activities have on the environment and local cultures of a region. The objective of this research is to analyze the contributions in the scientific and academic field of entrepreneurship in the tourism sector in the context of sustainability with the environment. The methodology used was descriptive-quantitative. Through a bibliographic analysis, the Web of Science (WOS) platform was chosen because it contains a high number of quality journals and impacts on the scientific community in the areas of social sciences, 69 were obtained. Articles in the main collection that represent open access documents with the search criteria "tourism entrepreneurship" AND "sustainable tourism". The main results revealed that the first article that links this topic arises from the year 2007 although it is since 2016 that interest in research has increased, focusing on the areas of green sustainable science technology and environmental studies. The main authors with scientific contributions correspond to Xavier Font and Marianna Sigala who focus their studies on sustainable marketing in tourism and tourist hospitality. England, Spain and Romania are the countries with the highest contributions.

The main conclusion determines that in European countries it is small companies that are more motivated to be sustainable, the vast majority of these enterprises carry out their activity in protected natural areas and believe that the support of public and private institutions is vital for the development of economic activities seeking the natural conservation of the planet.
\end{abstract}

Keywords: Entrepreneurship, tourism entrepreneurship, sustainable tourism

Artículo recibido: 02 noviembre. 2021 Aceptado para publicación: 28 noviembre 2021 Correspondencia: Ym.garcia.2016@alumnos.urjc.es

Conflictos de Interés: Ninguna que declarar 


\section{INTRODUCCIÓN}

El turismo es el sector económico líder a nivel mundial dado que representa un $10 \%$ del empleo mundial y un 10.4\% del PIB global (WTTC, 2018). El interés en los emprendimientos turísticos surge de la vinculación del espíritu empresarial con el entorno regional, nacional o local (Page \& Ateljevic, 2009). Los emprendimientos turísticos parten de la necesidad que tiene una población o región de satisfacer necesidades de turistas nacionales o extranjeros.

Por su parte, el turismo sostenible o turismo sustentable busca minorar el impacto que tienen las actividades turísticas al medioambiente y a las culturas locales de una región. El fin de las administraciones públicas es diversificar sus economías a través del turismo en sus regiones menos desarrollados, este punto de vista a generado debates a favor y en contra por considerarse "incentivo poco limpio" dañando ecosistemas naturales o afectando a las culturas de la zona. Sin embargo, sigue siendo una buena alternativa de crecimiento para los gobiernos (Loannides \& Debbages, 1997).

La principal atención sobre los emprendimientos turísticos radica en el impacto que tiene en la naturaleza física, biología, sociocultural y económica, que pueden ser positivas o negativas (Mathieson y Wall, 1982; Crick, 1992). El concepto de turismo sustentable surge a finales de los 80 y principios de los 90, considerado un nuevo modelo de desarrollo en donde intervienen el turismo de aventura, el turismo rural, turismo cultural y el ecoturismo (Tarlobani, 2005, p:224).

Dentro de la revisión de la literatura varios son los estudios que consideran la relación de sustentabilidad con el turismo, destacando asi factores que están relacionados con el inicio de una actividad empresarial. Entre los que se destacan (Vera et al., 1997):

- El crecimiento poblacional que conlleva al desarrollo de más empleos y empresas

- El concepto de conciencia verde

- Calidad ambiental en los paises sobretodo en los desarrollados

- El ecosistema turistico (turistas, población, gobiernos)

- Deterioro de destinos turisticos consolidados

El presente artículo tiene como objetivo analizar los aportes en el campo científico y académico de los emprendimientos en el sector turístico en el contexto de la sustentabilidad con el entorno a través de los indicadores bibliométrico utilizando la 
plataforma web of Science de Clarivate Thomson y la construcción de mapas científicos de la ciencia con el software VosViewer.

La presente investigación contribuye a la comunidad académica como fuente de información sobre los avances existentes en la temática.

Los resultados muestran los avances que está obteniendo los emprendimientos turísticos sustentables que en realidad es un tema que ha llamado su atención mundial desde finales de 1980 pero en el campo científico no es hasta la década pasada (2016) que presenta interés en los investigadores contando en la actualidad de 20 documentos en el año 2020. Por consiguiente, el artículo se divide en tres secciones, la primera de ella se presenta la introducción y metodología, en la segunda sección muestra los resultados hallados de forma cuantitativa y descriptiva y la última sección corresponde a las conclusiones y limitaciones del presente estudio.

\section{METODOLOGÍA}

Para realizar el presente estudio bibliométrico se utilizó la base de datos de referencias Web Of Science (en adelante W.O.S.) que contiene información de publicaciones científicas y periódicas desde 1900 hasta la actualidad y que abarca las áreas de: Ciencias, Ciencias Sociales y Arte y Humanidades, con más de 14.500 revistas de investigación de alto impacto con información adecuada para el análisis cuantitativo (Baier-Fuentes et al., 2018; Guerras-Martin et al., 2020) y herramientas de evolución como son el Journal Citation Report y Essential Science Indicators.

La Bibliometría permite evaluar y monitorear un campo especifico de la ciencia como puede ser demostrado a través del número de investigaciones existentes de un tema (Cobo, López-Herrera, Herrera-Viedma, \& Herrera, 2010).

Se realizó la búsqueda avanzada en la colección principal de W.O.S. con la etiqueta de campo tema en julio de 2021, utilizando los términos "tourism entrepreneurship" AND "sustainable tourism". Se consideraron tres criterios de inclusión: i) fecha: todos los periodos excluyendo el año 2021, ii) áreas de conocimiento: negocios, gestión y economía, iii) tipo de publicación: artículos y revisiones.

Del proceso detallado, se obtuvo 69 artículos de referencia para el análisis que permitió identificar los países, el número de artículos publicados desde 2007 donde se evidencia el primer trabajo sobre los factores determinantes del emprendimiento y las diez principales revistas con el mayor número de publicaciones. Para el análisis de los datos 
se utilizó el software de mapas de la ciencia: VosViewer que es un programa desarrollado para construir y visualizar mapas a partir de una base de datos bibliométrica (Van Eck y Waltman, 2014), las redes pueden enlazarse por coautoría, co-ocurrencia, citación, acoplamiento bibliográfico y co-citación considerando como unidad de análisis: los autores, las organizaciones y los países (Caputo et al., 2018; Mascarenhas et al., 2017). Para este artículo, se elaboró un mapa de co-autoria para autores, paises y organizaciones y co-ocurrencia para las palabras claves.

\section{RESULTADOS}

De un total de 69 artículos descargados de la plataforma Web of Science se presenta a continuación los resultados obtenidos.

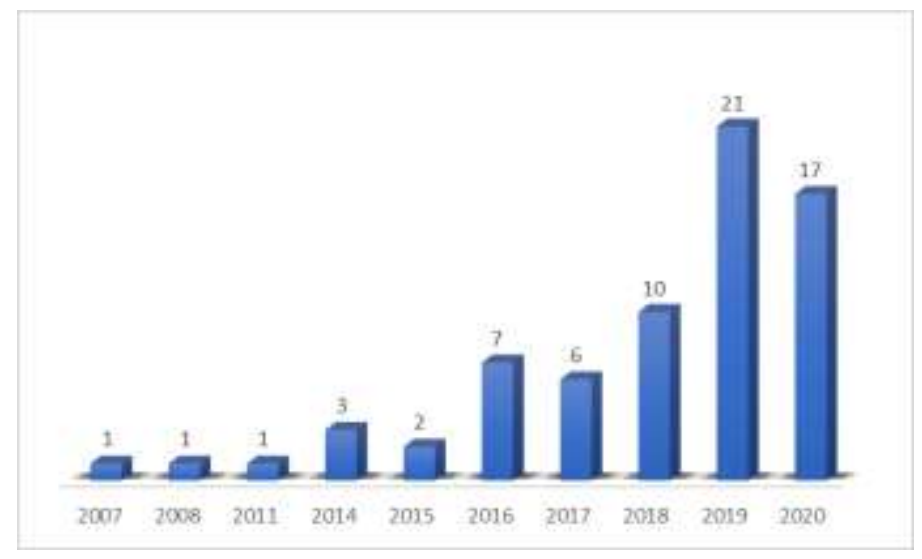

Figura 1 Publicaciones por año

Las investigaciones referentes a los emprendimientos turísticos sustentables empiezan a llamar la atención de la comunidad científica académica desde el año 2007 con un documento, pero es a partir del año 2016 que comienza a generar mayor interés con 7 publicaciones alcanzando el máximo de publicaciones en el año 2019 con 21 documentos.

Tabla 1 Top Ten Revista con mayores publicaciones

\begin{tabular}{lcc}
\hline Revista & Documentos & JCR \\
\hline Sustainability & 21 & $\mathrm{Q} 2$ \\
Journal of Sustainable Tourism & 8 & $\mathrm{Q} 1$ \\
Pasos Revista de Turismo y Patrimonio Cultural & 3 & --- \\
2nd. International Conference on Strategic Innovative Marketing & 2 & ---- \\
Asia Pacific Journal of Tourism Research & 2 & $\mathrm{Q} 3$ \\
International Journal of Contemporary Hospitality Management & 2 & $\mathrm{Q} 2$ \\
Procedia Social and Behavioral Sciences & 2 & ---- \\
Tourism Planning and Development & 2 & ---- \\
Baltic Journal of Economic Studies & 1 & ---- \\
Business System Research Journal & 1 & ---- \\
\hline
\end{tabular}


La tabla uno muestra las revistas que cuenta con el mayor número de documentos siendo las dos primeras de la lista top perteneciente al indicador JCR con Quartil dos y Quartil uno respectivamente. La revista sustainability es de campo multidisciplinar con publicaciones exclusivamente en inglés. Las revistas que no presenta indicador es porque no se encuentra dentro de la categoría del Indice JCR bien porque pertenece a Scopus o Latindex.

Tabla 2 Artículos con mayor número de publicaciones

\begin{tabular}{|c|c|c|c|c|}
\hline Articulo & Autores & Revista & Citas & Año \\
\hline $\begin{array}{l}\text { Sustainability motivation and } \\
\text { practices in small tourism } \\
\text { enterprises in European protected } \\
\text { areas }\end{array}$ & $\begin{array}{ll}\text { Font, } & \text { X.; } \\
\text { Garay, } & \text { L.; } \\
\text { Jone, S. } & \end{array}$ & $\begin{array}{l}\text { Journal of } \\
\text { Cleaner } \\
\text { Productions }\end{array}$ & 91 & 2016 \\
\hline $\begin{array}{l}\text { Sharing versus collaborative } \\
\text { economy: How to align ICT } \\
\text { developments and the SDGS in } \\
\text { Tourism? }\end{array}$ & $\begin{array}{l}\text { Gossling, } \\
\text { Hall, CM. }\end{array}$ & $\begin{array}{l}\text { Journal of } \\
\text { Sustainable } \\
\text { Tourism }\end{array}$ & 55 & 2019 \\
\hline $\begin{array}{l}\text { Opportunites for cross-border } \\
\text { entrepreneurship development in } \\
\text { a cluster model exemplified by } \\
\text { the Polish-Czech Border Region }\end{array}$ & $\begin{array}{l}\text { Kurowska- } \\
\text { Pysz, J. }\end{array}$ & $\begin{array}{l}\text { Sustainable } \\
\quad \text { Asia } \\
\text { Conference }\end{array}$ & 35 & 2016 \\
\hline $\begin{array}{l}\text { The role of self gentrification in } \\
\text { sustainable tourism: Indigenous } \\
\text { entrepreneurship at Honghre ham } \\
\text { rice terraces world heritage site, } \\
\text { China }\end{array}$ & $\begin{array}{ll}\text { Chan, } & \text { JH; } \\
\text { Iankova, } & \text { X.; } \\
\text { Zhang, } & \text { Y.; } \\
\text { McDonald, } & \text { T.; } \\
\text { Qi, XG. } & \\
\end{array}$ & $\begin{array}{l}\text { Journal of } \\
\text { Sustainable } \\
\text { Tourism }\end{array}$ & 26 & 2016 \\
\hline $\begin{array}{l}\text { Exploring Agritourism } \\
\text { Entrepreneurship in the UK. }\end{array}$ & $\begin{array}{l}\text { Phelan, C.; } \\
\text { Sharply, R. }\end{array}$ & $\begin{array}{c}\text { Tourism } \\
\text { Planning and } \\
\text { Development }\end{array}$ & 25 & 2011 \\
\hline $\begin{array}{l}\text { Exploring the antecedents of } \\
\text { sustainable tourism development }\end{array}$ & $\begin{array}{l}\text { Mackenzie, N.; } \\
\text { Gannan, MJ. }\end{array}$ & $\begin{array}{l}\text { International } \\
\text { Journal of } \\
\text { Contemporary } \\
\text { Hospitality } \\
\text { Management } \\
\end{array}$ & 24 & 2019 \\
\hline $\begin{array}{l}\text { Farm Diversification and } \\
\text { Sustainability of Multifunctional } \\
\text { Peri Urban Agricultura: } \\
\text { Entrepreneurial Attributes of } \\
\text { Advanced Diversification in } \\
\text { Japan }\end{array}$ & $\begin{array}{ll}\text { Yoshida, } & \text { S.; } \\
\text { Yagi, } & \text { H.; } \\
\text { Kiminami, } & \text { A.; } \\
\text { Garrod, G. } & \end{array}$ & Sustainability & 15 & 2019 \\
\hline $\begin{array}{l}\text { Sustainable Business Models of } \\
\text { SME's: Challenge in Yacht } \\
\text { Tourism sector }\end{array}$ & $\begin{array}{l}\text { Battistella, C.; } \\
\text { Cagnina, MR.; } \\
\text { Cicero, L.; } \\
\text { Preghenella, N. }\end{array}$ & Sustainability & 14 & 2018 \\
\hline
\end{tabular}




\begin{tabular}{|c|c|c|c|c|}
\hline $\begin{array}{lr}\text { Heritage } & \text { toruism } \\
\text { entrepreneurship and } & \text { social } \\
\text { media opportunities } & \text { and } \\
\text { challenges }\end{array}$ & $\begin{array}{l}\text { Surugui, MR.; } \\
\text { Surugui, C. }\end{array}$ & & 14 & 2015 \\
\hline $\begin{array}{l}\text { Heritage as an alternative driver } \\
\text { resilient for sustainable } \\
\text { development and economic } \\
\text { recovery in South East Europe }\end{array}$ & & & & \\
\hline 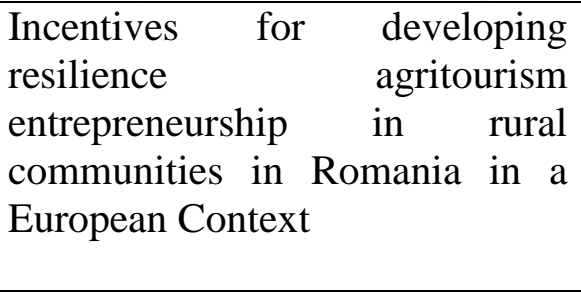 & $\begin{array}{l}\text { Dragoi, MC.; } \\
\text { Iamand, IE.; } \\
\text { Munteanus, } \\
\text { SM.; Ciobanu, } \\
\text { R., Tartavrilea, } \\
\text { RI.; Ladaru, R. }\end{array}$ & Sustainability & 13 & 2017 \\
\hline
\end{tabular}

La tabla 10 muestra los artículos con mayor número de citaciones referentes a la temática de estudio, el documento "Sustainability motivation and practices in small tourism enterprises in European protected áreas" con los autores Font, Garay y Jone publicado en el año 2016 cuenta con 91 citas. El artículo hace énfasis a las motivaciones que tienen las pequeñas empresas con actividades en las áreas protegidas de Europa.

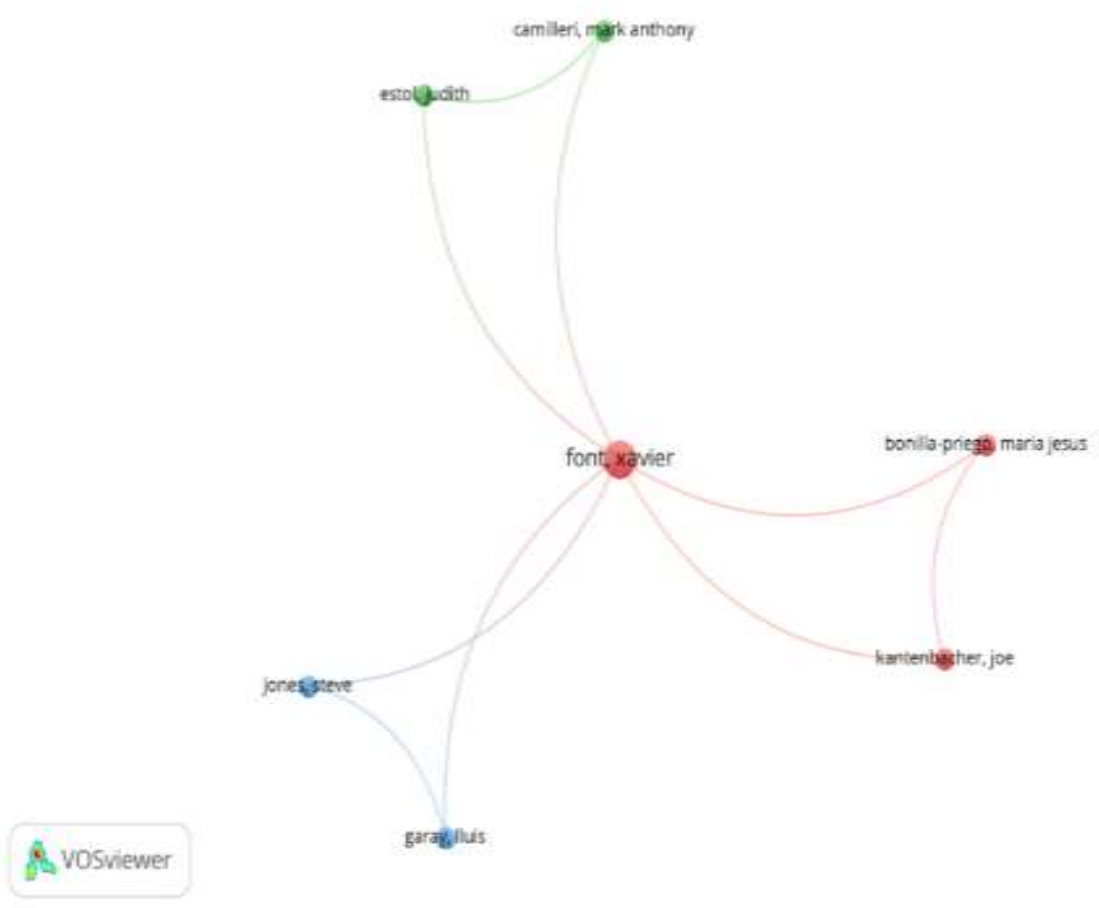

Figura 2 Mapa de coautoría de autores

En la figura dos muestra la relación de coautoría entre los principales autores, para mejorar la visualización del mapa se utilizó como filtro autores que cuenten con un mínimo de cinco citaciones. De un total de 205 autores, el software Vosviewer arrojo 
como resultado tres clústeres. El clúster uno (rojo) es considerado el de mayor relevancia al contener los principales investigadores de la temática que son: Bonilla-Priego, Font y Kantenbacher perteneciente a la Universidad Rey Juan Carlos, University of Surrey y University South Dakota respectivamente dedicados a realizar investigaciones referentes a hospitalidad turística, marketing turístico y consumo sustentable.

En el clúster dos (verde) aparecen Camilleri y Estol perteneciente a la Universidad de Malta y Barcelona respectivamente centran sus investigaciones al turismo responsable y economía circular.

En el clúster tres (azul) aparecen Garay y Jones perteneciente a la Universidad Oberta de Catalunya y Leeds Metropolitan University respectivamente sus investigaciones se enfocan en sustentabilidad de las pequeñas empresas y narrativas turísticas.

vosviewer

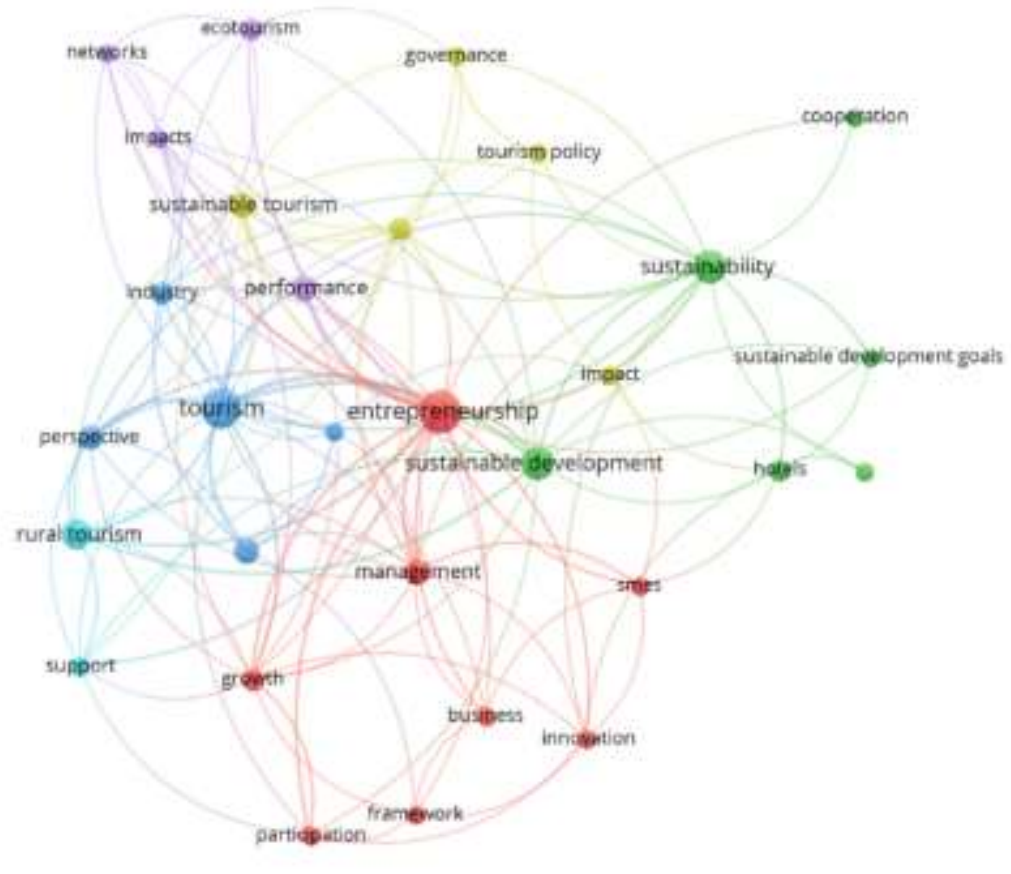

Figura 3 Palabras claves

La figura 10 muestra la co-ocurrencia de las palabras claves tomando como base aquellos documentos que contienen como mínimo la relación de tres palabras claves. De un total de 468 palabras claves se obtuvieron seis clústeres.

Clúster uno (rojo): aparecen las palabras business, entrepeneurship, framework, growth, innovation, management, participation, Smes. Clúster dos (verde): Cooperation, hotel, sustainability, sustainable, development, goals, tourism entrepreneurship. Clúster tres ( 
azul ): Industry, perspective, social entrepreneurship,, sustaibanle entrepreneurship. Clúster cuatro (amarillo): Competitiveness, governance, impact, sustainable tourism. Clúster cinco (lila ): ecoturism, impacts, network, performance. Clúster seis (celeste): rural tourism, support.

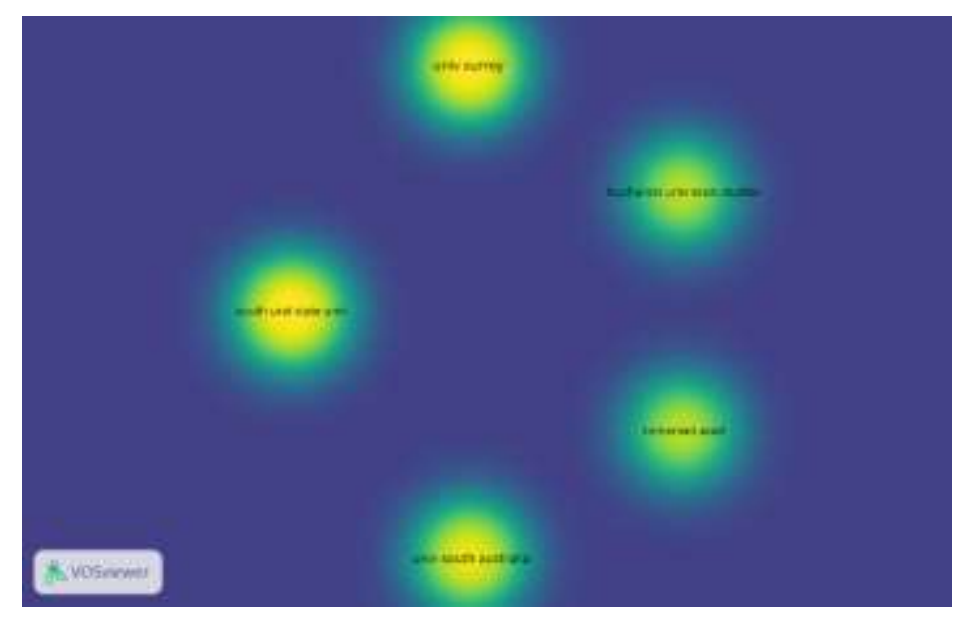

Figura 4 Organizaciones

La figura 4 muestra la colaboración entre organizaciones referentes a la temática de estudio, para mejorar la visualización del mapa se usó como filtro las organizaciones que tienen como mínimo dos documentos y cinco citaciones. Se ha obtenido cinco clústeres. Clúster uno: South Ural, Un. Novi, Clúster dos: Bucharest, Cluster tres: Romanian, Cluster cuatro: South Australia, Cluster cinco: University Surrey

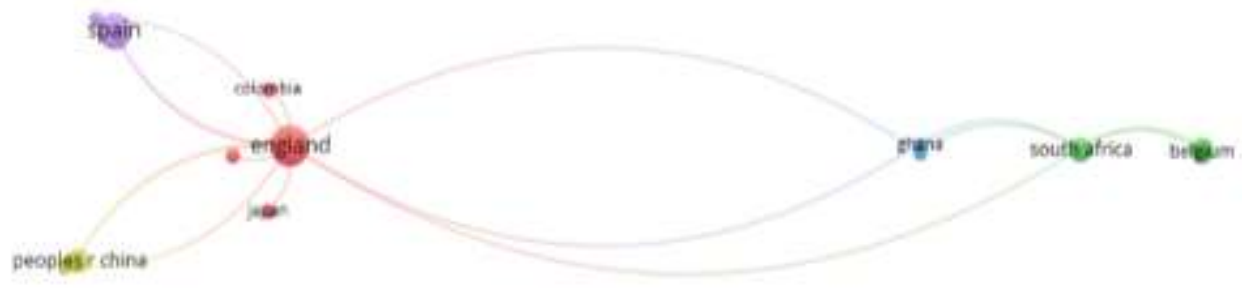

voswewer

Figura 5 Países

La figura 5 muestra la colaboración entre Paises. De un total de 47 países se obtuvieron cinco clústeres. Clúster uno: Colombia, Inglaterra, Gambia y Japón, Clúster dos: Bélgica, Netherland, South Africa, Clúster tres: Ghana, Nigeria, Clúster cuatro: China, Arabic Saudit, Cluster cinco: Malta, España. 


\section{CONCLUSIONES}

El turismo sustentable a pesar de presentarse dentro de la revisión de la literatura documentos encontrados desde finales de los años 80, en el mundo científico académico cobra interés a partir del año 2007. Solo 69 documentos fueron analizados en el presente artículo que hacen referencia a los emprendimientos turísticos enfocados en la sostenibilidad y sustentabilidad del ecosistema. El turismo depende de la cantidad y calidad de emprendimientos que subsistan a largo plazo a través de la sostenibilidad (Butkouskaya, Romagosa, \& Noguera, 2020). Al igual que el estudio realizado por Crnogaj et al. (2014) en el presente artículo se evidencia los escasos investigaciones científicas que relacionan el emprendimiento, el turismo y la sustentabilidad.

La mayoría de los emprendimientos turísticos sustentables son formados por las pymes, las mismas que son representadas por mujeres (Bosma et al., 2019; Johansen, 2013). Estos emprendimientos son realizados en las areas rurales de los paises buscando sobretodo el equilibrio entre lo economico y el bienestar. El termino más familiarizado para este tipo de emprendimientos es emprendimiento social.

La mayoria de investigaciones son de tipo empirico realizados en su gran mayoria en Europa, solo dos paises de sudamerica ha vinculado este tipo de estudio en sus regiones como son: Brasil y Colombia.

Las limitiaciones del estudio se enfocan en el uso de una solo plataforma de descarga de los documentos como la WOS y la filtración de los mismo a estudios de acceso abierto y el area de negocios y economia.

Las futuras lineas de investigación debe ampliarse a otras zonas o regiones de estudios, la comparativas por género y la implicación de los gobiernos e instituciones educativas sobre el fomento de emprendimientos sustentables y sostenibles.

\section{REFERENCIAS BIBLIOGRAFICAS}

Baier-Fuentes, H., Cascón-Katchadourian, J., Sánchez, Á., Herrera-Viedma, E., \& Merigó, J. (2018). A bibliometric overview of the international journal of interactive multimedia and artificial intelligence. International Journal of Interactive Multimedia and Artificial Intelligence.

Bosma, N., Schott, T., S.A., T., \& Kew, P. (2019). Special Topic Report: Social Entrepreneurship. Global Entrepreneurship Monitor (GEM). 
Butkouskaya, V., Romagosa, F., \& Noguera, M. (2020). Sustainability obstacles to sustainable entrepreneurship amongst torism students. A Gender Comparison. Sustainability.

Caputo, A., Marzi, G., Pellegrini, M., \& Rialt, R. (2018). Conflict Management in Family Businesses: A bibliometric analysis and systematic literature review. International Journal of Conflict Management 29 (2).

Cobo, M., López-Herrera, A., Herrera-Viedma, E., \& Herrera, F. (2010). An approach for detecting, quantifying and visualizing the evolution of a research field: A practical application to the fuzzy sets theory field. Journal of Informetrics, 146-166.

Crick, M. (1992). Representaciones del turismo internacional en las Ciencias Sociales. Los mitos del turismo Endymion, 341-403.

Crnogaj, K., Rebernik, M., Bradac Hojnik, B., \& Onrerzel Gomezelj, D. (2014). Building a model of reseraching the sustainable entrepreneurship in the tourism sector. Kybernetes.

Guerra-Martin, L., Ronda-Pupo, G., Zúñiga-Vicente, J., \& Benito-Osorio, D. (2020). Half a century of research on corporate diversification: A new comprehensive framework. Journal Business Research.

Johansen, V. (2013). Entrepreneurship education adn start-up activity. A gender perspective. International Journal Gender Entrepreneruship , 216-231.

Loannides, D., \& Debbages, K. (1997). Economic geographic and tourism. Routlegde New York.

Mascarenhas, C., Marques, C., \& Galvao, A. (2017). Entrepreneurial university towards a better understanding of past trends and future directions. Journal of Enterprising Communities People and Places in the Global Economy.

Mathieson, A., \& Wall, P. (1982). Torism, Economic, physical and social impacts. Longman, London/NewYork.

Page, C., \& Ateljevic, J. (2009). Tourism and Entrepreneurship: International Perspective. Oxford: Butterworth Heinemann.

Tarlombani, M. A. (2005). Turismo y sustentabilidad: Entre el discurso y la acción. Estudios y perspectivas en Turismo, 222-242.

Van Eck, N. J., \& Waltman, L. (2014). Visualizing Bibliometric Networks. . Measuring Scholarly Impact, 285-320. 
Emprendimientos turísticos sustentables...

Vera, F. e. (1997). Análisis Territorial del turismo. Una nueva geografía del turismo. Ariel, Barcelona.

WTTC. (2018). World Travel and Tourism Council Economic Impact. London: WTTC. 